\title{
Adaptive Image Enhancement Algorithm Based on Matching Pyramid Decomposition
}

\author{
Linlin Zhao \\ School of Automation and Electrical Engineering, Lanzhou Jiaotong University, Lanzhou 730070, \\ China. \\ Zhaolllzjtu@163.com
}

Keywords: Image enhancement, matching pyramid decomposition, feature points, adaptive enhancement.

\begin{abstract}
Image enhancement technology is targeted to highlight the overall and local useful information characteristics of the images, expand the differences between different feature points in the image, rich image information to meet the application needs of different environments. The current image enhancement algorithm uses corner point extraction and feature matching technology, information loss and distortion can occur during SIFT feature point matching, the performance is not good. An image enhancement algorithm based on adaptive projection matching and pyramid decomposition is proposed, feature extraction and multi-layer purification of blurred images are performed on the scale translational plane, the discrete dopplerlet transform is used to reconstruct the image, the high frequency image is denoised by adaptive matching projection filtering, and the grey pixel value of image was enhanced by adaptive pyramidal matching projection decomposition. Simulation results show that the proposed algorithm is effective to remove or reduce the noise, and the image enhancement effect is good, and the peak signal-to-noise ratio is improved.
\end{abstract}

\section{Introduction}

With the vigorously popularizing of the sensor technology and computer science technology, computer graphics and image processing technology as a kind of advanced data information transmission and processing technology is developed in recent years. Through the computer digital image processing, useful features in the image can be effectively discovered, and the edge of the image target and detail characteristics can be accurately extracted, as a result, the image recognition ability is improved. Modern multimedia digital image communication and signal processing cannot leave without the image processing and the related algorithm design, through image compression, coding, enhancement and smoothing, the detailed analysis and recognition ability of the image are improved, to achieve image optimal transmission and storage, etc. Image enhancement technology is an important branch of image processing. Problems such as underexposed and overexposed are usually emerged in the blurred image acquisition, which lead to useful information loss or distortion, through image enhancement, it can improve the imaging visual effect, purposefully stresses global or local characteristics of image, improves image quality, and enriches information, therefore, image enhancement is of great significance in areas such as image recognition and image detail feature extraction $^{[1-3]}$. Through the image enhancement algorithm design, targeted to highlight the overall and local useful information feature of the image, expand the differences between different feature points in the image, and enrich image information, these will make the image texture structure and the detailed characteristics strengthened, so as to strengthen the image interpretation and recognition effect, and meet the application requirements of different environments.

Image enhancement can be divided into two categories: frequency domain method and spatial domain method. Frequency domain method models the image as a two-dimensional signal, uses the time-frequency characteristic analysis and fourier transform method to realize a image low pass filter. Filter out high dimensional vector characteristics of image, achieve image enhancement. Spatial domain method directly do operation on the image grayscale, it is an indirect enhancement algorithm. 
Space domain algorithm was divided into the point-operation algorithm and neighborhood de-noising algorithm $^{[4-6]}$. In traditional methods, the study of image enhancement algorithm has obtained certain achievements. Literature [7] puts forward an image enhancement technology based on twodimensional fourier transform, and time frequency analysis based on two-dimensional fourier transform is taken to make the original blurred image to be clear, highlights the characteristics of the image differences, however, the algorithm cannot effectively enhance image texture details. Literature [8] presents an image enhancement algorithm based on third order PDE subspace noise reduction, the original image is first processed with the gaussian filter through certain means, image enhancement is realized by using piecewise linear regression fitting method, improves the display ability of the image details, but in the high-dimensional space the algorithm needs large amount of calculation and has bad real-time performance. In addition, literature [9] uses an image enhancement technology based on angular point extracting and feature matching method, while the information loss and distortion can occur during SIFT feature point matching, and the performance is bad ${ }^{[10-13]}$.

Aiming at above problems, this paper uses the nonlinear time-varying characteristics of the discrete Dopplerlet transform, proposes an image enhancement algorithm based on adaptive matching projection and pyramid decomposition. In this paper, the feature extraction and multi-layer purification treatment is made for fuzzy image in the scale translations plane, and the discrete Dopplerlet transform is adopted to reconstruct the time-frequency image, through the adaptive matching projection filtering to denoise high frequency image, and then, using adaptive matching projection pyramid decomposition to enhance the gray pixel values of the image. Performance have been verified by carrying out simulation experiments, the results show the superior performance of the proposed adaptive image enhancement algorithm, and reaches effective conclusions.

\section{Image Feature Extraction and Multilayer Evolution Process}

\subsection{Feature Information Model Building and Information Extraction of Blurred Image.}

Image enhancement is to make original blurred image become clear or emphasize useful features. Based on the signal processing algorithm, the article models the image as a two-dimensional signal, and enhance the signal based on two-dimensional fourier transform. Where, $\mathrm{H}$ for the Hilbert space, define $D=\left(d_{\gamma}\right)_{\gamma \in \Gamma}$, is random distribution space for the image in the two-dimensional plane, if $\forall a, b \in R, a<b$, define $C([a, b], R)$ is basis function set $R$ in image characteristics pixel frequency range $[a, b], I(x, y)$ denotes the image grey value at $(x, y)$, the two-dimensional signal model of images based on time-frequency analysis method is as follow:

$$
G(x, y, \sigma)=\frac{1}{2 \pi \sigma^{2}} e^{-\frac{\left(x^{2}+y^{2}\right)}{2 \sigma^{2}}}
$$

To determine whether the image pixel is the angular point, the scale extremum value of two dimensional pixel image is used to detect the nonlinearity, its matrix form is as follow:

$$
D=\left[\begin{array}{cc}
I_{x}^{2} & I_{x} I_{y} \\
I_{x} I_{y} & I_{y}^{2}
\end{array}\right]
$$

Assuming that $\alpha$ denotes a horizontal displacement of the target image, $\beta$ denotes the zoom factor, the ratio between $\alpha$ and $\beta$ is $\gamma$, mean $\alpha / \beta=\gamma$, the functional relationship is as follow:

$$
\frac{\operatorname{det}(D)}{\operatorname{trace}^{2}(D)}=\frac{\alpha \cdot \beta}{(\alpha+\beta)^{2}}=\frac{\gamma}{(\gamma+1)^{2}}
$$

Calculating the peak crest trajectory of frequency domain image signal, it can prove that, for the scale $\alpha$ which is very close to 1 , wavelet peak crest trajectory of image frequency domain signal is as follow:

$$
a=1-\frac{B}{T f_{0}} b
$$


After angular point wavelet ridges of continuous image pixels are extracted, for frequency domain signal, their wavelet ridges have straight trajectory characteristics similar to the image frequency domain characteristics when their scales near to 1 , using the characteristic value as the weighted principal component to realize image recognition, the characteristic information measure space parametric equation of the blurred image is:

$$
\left(\begin{array}{l}
x_{1} \\
y_{1}
\end{array}\right)=k \cdot\left(\begin{array}{cc}
\cos \theta & \sin \theta \\
-\sin \theta & \cos \theta
\end{array}\right)\left(\begin{array}{l}
x \\
y
\end{array}\right)+\left(\begin{array}{c}
\Delta x \\
\Delta y
\end{array}\right)
$$

where, $\Delta x$ and $\Delta y$ represent horizontal displacement and vertical displacement of image angle feature, $k$ represents the image convolution factor, and $\theta$ represents gaussian difference displacement. Through software programming, to realize the output of the image motion parameters $\Delta x, \Delta y, k$ and $\theta$. According to the above processing, the two-dimensional signal model of image is built, in the gray histogram of the image, gray sliders with three angles traverse the circular area, image stratified ${ }^{\text {[14-17], }}$ to layered purify and enhance the image, the process description of feature key points expression in the image histogram is shown in figure 1.

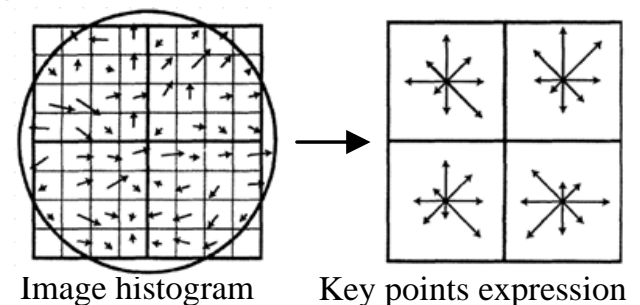

Fig. 1 Process description of feature key points expression in image histogram

\subsection{Image Multi-layer Purification Pretreatment Based on Time-frequency Analysis.}

In the process of image enhancement, the high frequency parts of blurred images need to be filtered and smoothed, to realize the image of multipath purification, and to improve image visual effect. In this paper, image purification processing is performed by using time-frequency analysis method based on wavelet decomposition, the wavelet function is:

$$
\begin{aligned}
& S_{g i v}(x, y)=-\log \left(P_{i v}(x, y)\right) \\
& S_{g i}(x, y)=S_{g i f}(x, y)+S_{g i v}(x, y)
\end{aligned}
$$

where, $G\left(x, y, \sigma_{i}\right)$ represents multipath interference distribution function, $\sigma_{i}$ is the Rayleigh differential scale is image acquisition process, and $w_{i}$ is the size of time delay window. The detail of the image is decomposed by continuous wavelet transform, and the continuous wavelet transform is:

$$
W_{\psi} y(a, b) \leq y, \psi_{a, b} \geq \int_{-\infty}^{+\infty} y(t) \frac{1}{\sqrt{|a|}} \psi^{*}\left(\left(\frac{t-b}{a}\right) \mathrm{d} t\right)
$$

Frequency modulation rule of blurred image data signal is hyperbolic frequency modulation, namely:

$$
f_{i}(t)=\frac{K}{t_{0}-t} \quad|t| \leq \frac{T}{2}
$$

After translation process in time domain, choose the time window width, and get the image data signal amplitude information, namely:

$$
u_{a}\left(t-b_{m}\right)=\frac{1}{\sqrt{a}} u(t) \quad-\frac{a T}{2}+b_{m}<t<\frac{a T}{2}+b_{m}
$$

In the wavelet frequency domain, it is easy to cause the edge blurred, by scale translation changes, the image characteristic data mining results at the point $\left(a, b_{m}\right)$ in the scale translation plane can be got:

$$
\begin{aligned}
W_{u} u\left(a, b_{m}\right) & =\frac{1}{\sqrt{a}} \int_{T / 2}^{T / 2} u(t) u^{*}\left(\frac{t-b_{m}}{a}\right) \mathrm{d} t \\
& =\frac{1}{\sqrt{a}} \int_{-a T / 2+b_{m}}^{T / 2} u(t) u^{*}(t) \mathrm{d} t=\frac{1}{\sqrt{a}} \int_{-a T / 2+b_{m}}^{T / 2}|u(t)|^{2} \mathrm{~d} t
\end{aligned}
$$

Thinking the detailed features $f(t)$ of the original image as the mother wavelet function $\psi(t)$, and using the wavelet packet method to calculate time scale factor of mother wavelet function, then get: 


$$
f_{s, \tau}(t)=[U(1 / s, \tau) f(t)]=\sqrt{|s|} f(s(t-\tau))
$$

Adopting wavelet basis function matching doppler image signal, then after wavelet packet layered purification, the image pixel output is as follow:

$$
\theta(t)=2 \pi \int_{-T / 2}^{t / a}\left(\frac{K}{t_{0}-t^{\prime}}\right) \mathrm{d} t^{\prime}=-2 \pi K \ln \left(1-\frac{t}{a t_{0}}\right)+\theta_{0}
$$

Through the grayscale correction in the histogram, and expand the dynamic range images, all these provide a clean image pixels feature for the purpose of image enhancement.

\section{Image Adaptive Matching Projection Pyramid Decomposition and Enhancement Algorithm Implementation}

\subsection{Image Time-frequency Reconstruction and Adaptive Matching Projection Filtering}

On the basis of the multi-layer purification pretreatment based on time-frequency analysis, the image time-frequency reconstruction and adaptive matching projection filtering are performed to improve the image enhancement algorithm. Analyzing the traditional methods, it shows that using the image enhancement technology with angular point extraction and feature matching, the information is easy to be lost and distorted in the SIFT feature point matching process, and the performance is poor. In order to overcome the disadvantages of traditional methods, this paper proposes an image enhancing algorithm based on adaptive matching projection and pyramid decomposition, the discrete dopplerlet transform is used for time-frequency image reconstruction. Reconstruction process description is as follows: For image frequency domain signal, the stationary observer is perpendicular to the direction of the source movement, considering the scalability of image signal decomposition scale, and choosing Doppler signal as the basis function, the multi-layer purification results after image time-frequency analysis is as follows:

$$
W_{u} u\left(a, b_{m}\right)=\frac{1}{\sqrt{a}} \int_{-a T / 2+b_{m}}^{T / 2}\left|\frac{1}{\sqrt{T}}\right|^{2} \mathrm{~d} t=\frac{1}{\sqrt{a} T}\left(\frac{T}{2}+\frac{a T}{2}-b_{m}\right)
$$

where, $T$ is the image sampling time center, $a$ is the neighborhood pixel gray value and $b_{m}$ is the low frequency characteristic parameters. The time-frequency sampling and high frequency suppression of image are carried through normalized amplitude modulation, and the unified form of image pixel characteristics of output is as follows:

$$
W_{u} u\left(a, b_{m}\right)=\frac{1}{\sqrt{a}}\left(\frac{a+1}{2}+\frac{|a-1|}{B / f_{0}}\right)
$$

where, $f_{0}$ is reference frequency, $B / f_{0}$ is the center spectrum point. The transformation coefficient value is discretized in some transformation domain of the image, and the instantaneous frequency of the image frequency domain signal is expanded into series:

$$
\left\{\begin{array}{l}
f_{i}(t)=\frac{K}{t_{0}-t}=\frac{K / t_{0}}{1-t / t_{0}}=\frac{f_{\max } f_{\min }}{f_{0}}\left(1+\frac{t}{t_{0}}+\frac{t^{2}}{t_{0}^{2}}+\cdots\right) \\
|t| \leq \frac{T}{2}
\end{array}\right.
$$

Obviously, the first order approximation of image frequency domain signal is the result representation of image frequency domain spectrum analysis. The frequency domain transformation formula of image processing is as follows:

$$
\mathfrak{R}(\rho, \theta)=\int_{-\infty}^{\infty} \int_{-\infty}^{\infty} g(x, y) \delta(\rho-x \cos \theta-y \sin \theta) \mathrm{d} x \mathrm{~d} y
$$

where, $\delta(\cdot)$ is Delta function. By substituting the multidimensional results of wavelet transform, the transformation of image processing is:

$$
\mathfrak{R}_{W}(\rho, \theta)=\int_{-\infty}^{\infty} \int_{-\infty}^{\infty}\left|W_{f} r(a, b)\right| \delta(\rho-a \cos \theta-b \sin \theta) \mathrm{d} a \mathrm{~d} b
$$


Through above processing, the reconstruction of image in time-frequency space is realized, and the harmonic is modulated with a window function, which enhances the detail enhancement ability of the image.

\subsection{Image Enhancement Algorithm Implementation}

The high frequency image is denoised by adaptive matching projection filtering, and the adaptive matching projection pyramid decomposition is used to adaptively enhance the grey pixel value of the image. Define a continuous dopplerlet transform for a square integrable image pixel feature points $s(t)$ :

$$
\begin{aligned}
D T_{s(t)} & =\left\langle s(t), d_{t_{0}, \Delta_{t}, l, v, c}(t)\right\rangle \\
& =\int_{-\infty}^{+\infty} s(t) d_{t_{0}, \Delta_{t}, l, v, c}^{*}(t) \mathrm{d} t
\end{aligned}
$$

where, $\Delta_{t} \in \mathfrak{R}^{+}, h>0, v>0, c \in \mathfrak{R}^{+}$, “ $\langle\cdot\rangle$ ”denotes inner product, command $f \in H, d_{\gamma_{0}} \in D$, then the adaptive matching vector set $f$ of the image can be broken down into:

$$
f=\left\langle f, d_{\gamma_{0}}\right\rangle d_{\gamma_{0}}+R_{f}
$$

where, $\left\langle f, d_{\gamma_{0}}\right\rangle d_{\gamma_{0}}$ is the projection of the image characteristics in $d_{\gamma_{0}}$ direction, based on the adaptive matching projection, a framework of the basis function set is constructed to realize image enhancement. and the feature matching degree of image enhancement is determined by the following formula:

$$
\lambda^{n}\left(d_{\gamma_{0}}\right)=\int_{-\infty}^{+\infty} f(t) \cdot d_{\gamma_{0}}^{*}(t) \mathrm{d} t
$$

Through a number of best base searches, find the largest value $\lambda^{n}\left(d_{\gamma_{0}}\right)$, to determine the useful information $f(t)$ of the image, and make the original unclear image to be clear. According to the above analysis, the image enhancement algorithm is improved as follows:

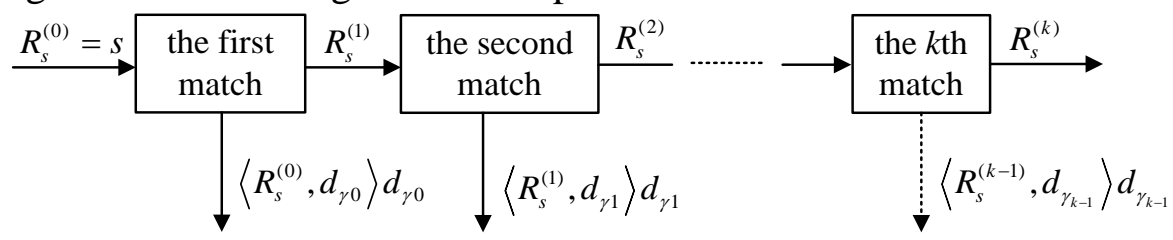

Fig. 2 Adaptive matching projection pyramid decomposition algorithm

\section{Image Enhancement Simulation Experiment and Performance Analysis}

To test the performance of this algorithm in the realization of image enhancement, the simulation experiment is carried out. The experimental simulation environment is: Intel (R) Core (TM) with 2 G memory, DDR2, the operation system is Windows 7, the simulation software uses Matlab R2009b. The original acquisition images as shown in figure 3.

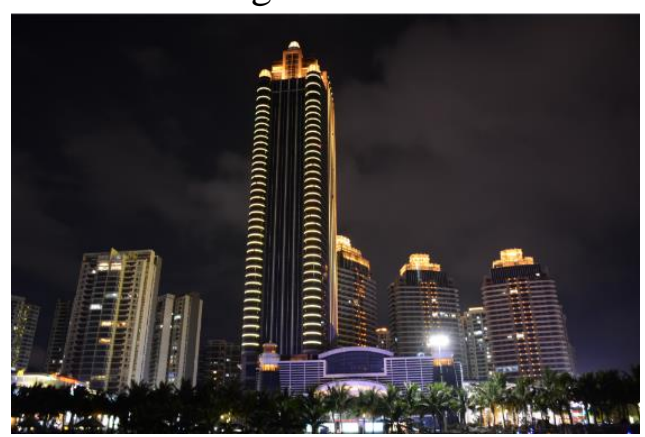

Fig. 3 The original image to be enhanced

Figure 3 shows that the detail characteristics such as lighting, clouds and other details in original night landscape are affected by high frequency interference effect, make the levels not strong, some unwanted image features cover important features, so the image enhancement processing is required.

Using the algorithm of this paper, the image is regarded as a two-dimensional signal, and the signal enhancement is carried out based on the two-dimensional fourier transform. The feature extraction and multi-layer purification of the blurred images are performed on the scale translational plane, Then the 
pixel values of the image are self-adapted to be enhanced using the matching tower decomposition in this paper, and get the image enhancement results. To compare the performance of the algorithm with the gradient method and mask matching method, the simulation results are shown in fig.4. It can be seen from the figure, using image enhancement algorithm in this paper, the influence of the noise has been effectively removed or reduced, the gradation of the cloud in the sky is outstanding, the images become clear, while the traditional methods of image smoothing either excessive, or the image sharpening excessively, lead to image enhancement effect is not good, the performance is inferior to method in this paper.

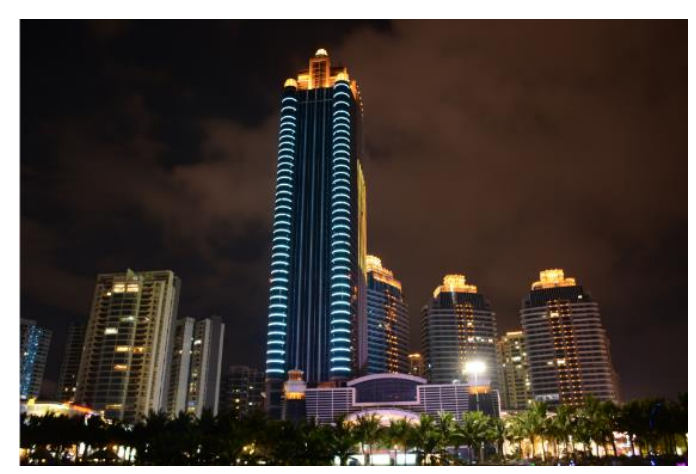

(a) Algorithm in this paper

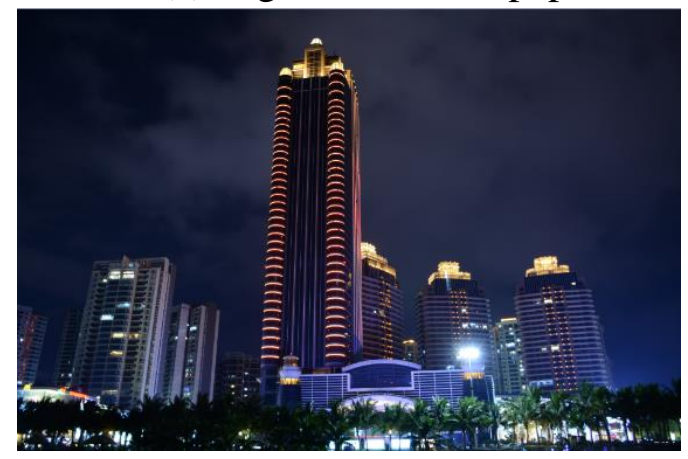

(b) Gradient method

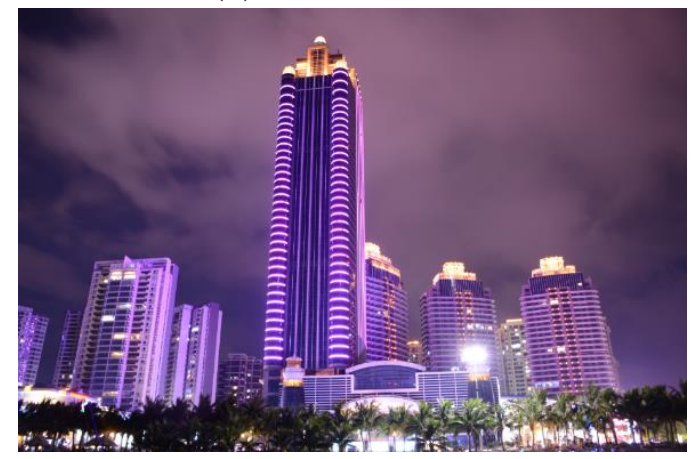

(c) Mask matching method

Fig. 4 Image enhancement output

In order to quantitatively analyze the performance and enhancement effects of the algorithm in this paper, the image is enhanced by means of the algorithm in this paper, gradient method and mask matching method, and the mean value, standard deviation and information entropy of image pixels are used to reflect the image brightness, contrast and the amount of information after image ehancement by three method, the results are shown in table 1.

Table 1. Image quality assessment metric

\begin{tabular}{ccccc}
\hline Evaluation index & $\begin{array}{c}\text { Original } \\
\text { image }\end{array}$ & $\begin{array}{c}\text { Mask matching } \\
\text { method }\end{array}$ & $\begin{array}{c}\text { Gradient } \\
\text { method }\end{array}$ & $\begin{array}{c}\text { Method in } \\
\text { this paper }\end{array}$ \\
\hline Mean values & 42.767 & 67.841 & 92.142 & 79.932 \\
Standard deviation & 37.443 & 56.849 & 60.315 & 73.538 \\
Information entropy & 5.993 & 8.028 & 7.582 & 7.203 \\
\hline
\end{tabular}


It can be seen from table 1: the mean value and the standard deviation of the original images are small, which indicates that original image is darker, and weaker contrast. The standard deviation of the original image is the largest using the method proposed in this paper, but the average value fell, shows that the contrast of the enhanced image increased obviously. Using gradient method to enhance the image, the mean value and standard deviation are increased obviously, which indicates that the brightness of the image is improved, while the mean value and standard deviation of the enhanced image after the mask matching method have not changed very much, shows that the enhancement effect is not good.

Comparing image information entropy after image enhancement using three method, it can be seen that by the algorithm in this paper the image information entropy closest to the original image, shows that the information is lost at least when the image is enhanced through the algorithm in this paper.

\section{Conclusion}

In the blurred image acquisition, usually produce the problem such as underexposed and overexposed, which will lead to useful information loss or distortion, through the image enhancement, improve image visual effect, purposefully stressed global or local characteristics of image, improve the image quality, enrich the information, An image enhancement algorithm based on adaptive matching projection and tower decomposition is proposed. Firstly, feature extraction and multi-layer purification of blurred images are performed on the scale translational plane, the discrete dopplerlet transform is used for image time-frequency reconstruction, the high frequency image is denoised by adaptive matching projection filtering, and adaptive matching projection tower decomposition is used to enhance the gray pixel value of image. The experimental results show that the algorithm for image enhancement processing in this paper, the influence of the noise effectively been removed or reduced, in this paper, from the nighttime images, the night in the sky clouds of gradation analysis, The layered analysis of clouds in the night sky is better, and the image becomes clear, the image enhancement effect is good, the peak signal-to-noise ratio increased, and these are useful for image feature extraction and recognition.

\section{References}

[1]. Jiang Chenglong, Zhang Bingchen, Wang Zhengdao, et al. Group-sparse complex approximate message passing algorithm for wide angle synthetic aperture radar imaging. JEIT. Vol. 37 (2015), No. 8, p. 1793-1800.

[2]. Liu Yanli, Gui Zhiguo. Adaptive image enhancement algorithm with variable weighted matching based on morphology. Vol. 36 (2014) No. 6, p. 1285-1291.

[3]. Wang Jingjing, Jia Zhenhong, Qin Xizhong, et al. Remote sensing image enhancement algorithm based on Shearlet transform and multi-scale Retinex. Journal of Computer Applications. Vol. 35 (2015) No. 1, p. 202-205.

[4]. Cetin M, Stojanovic I, Onhon O, et al. Sparsity-drivensynthetic aperture radar imaging: reconstruction, autofocusing, moving targets, and compressed sensing. IEEE Signal Processing Magazine. Vol. 31 (2014) No. 4, p. 27-40.

[5]. Luo Jun, Wang Lian, Hou Yan, et al. Sub-pixel corner detection of tessellated picture based on camera calibration. Journal of Chongqing University. Natural Science Edition, Vol. 31 (2008) No.6, p. 615-618.

[6]. Bai Meng, Li Minhua, LÜ Yingjun. Corner detection method of chess board based on symmetry analysis. Information and control. Vol. 44 (2015) No. 3, p. 276-283,290.

[7]. Wang Weihong, Yan Qin, Jin Dandan, et al. Object-oriented remote sensing image Classification Based on GEPSO Model. Computer Science, 2015, 42 (5):51-53, 71. 
[8]. Wu Jiexuan, Chen Zhenjie, Zhang Yunqian, et al. Utilizing multi-core CPU to accelerate remote sensing image classification based on K-means algorithm. Journal of Computer Applications. Vol. 35 (2015) No. 5, p. 1296-1301.

[9]. Li Pengwei, Ge Wenying, Liu Guoying. Shadow detection method based on shadow probability model for remote sensing images. Journal of Computer Applications. Vol. 35 (2015) No. 2, p. $510-514$.

[10]. Yang Leilei, Chen Songcan. Worst-separated couple-resolution discriminant analysis. Journal of Software. Vol. 26 (2015) No. 6, p. 1386-1394.

[11]. Yang C, Goodchild M, Huang Q, et al. Spatial cloud computing: how can the geospatial sciences use and help shape cloud computing. International Journal of Digital Earth. Vol. 4 (2011) No. 4, p. 305-329.

[12]. Xu Miaozhong, Cong Ming, Wan Lijuan, et al. A methodology of image segmentation for high resolution remote sensing image based on visual system and markov random field. Acta Geodaetica et Cartographica Sinica. Vol. 44 (2015) No. 2, p. 198-205.

[13]. Huang Weiguo, Gu Chao, Shang Li, et al. Hierarchical representation method for object recognition. Chinese Journal of Electronics. Vol. 43 (2015) No.5, p. 854-861.

[14]. Huang Wenming, Cai Wenzheng, Deng Zhenrong. Fast recognition model for cerebrospinal fluid images based on sparse coding. Journal of Computer Applications. Vol. 34 (2014) No. 7, p. 2040-2043.

[15]. Wang Wenbo, Yin Hong, Xie Wenbin, et al. Terrain rendering for level of detail based on hardware tessellation. Journal of Computer Applications. Vol. 35 (2015) No. 6, p. 1716-1719.

[16]. Javier Barrachina, Piedad Garrido, Manuel Fogue, et al. VEACON: A vehicular accident ontology designed to improve safety on the roads. Journal of Network and Computer Applications. Vol. 35 (2012) No. 6, p. 1891-1900.

[17]. Ma Xiaomin, Zhang Jinsong, Yin Xiaoyan, et al. Design and analysis of a robust broadcast scheme for VANET safety-related services. IEEE Transactions on Vehicular Technology. Vol. 61 (2012) No. 1, p. 46-61. 